\title{
Neuroprotective actions of estradiol revisited
}

\author{
Iñigo Azcoitia ${ }^{1}$, Maria-Angeles Arevalo ${ }^{2}$, Alejandro F. De Nicola ${ }^{3}$ and \\ Luis M. Garcia-Segura ${ }^{1}$
}

\author{
${ }^{1}$ Cell Biology, Faculty of Biology, Complutense University of Madrid, E-28040 Madrid, Spain \\ ${ }^{2}$ Instituto Cajal, CSIC, Avenida Doctor Arce 37, E-28002 Madrid, Spain \\ ${ }^{3}$ Laboratory of Neuroendocrine Biochemistry, Instituto de Biologia y Medicina Experimental-CONICET, Obligado 2490,1428 \\ Buenos Aires, Argentina
}

\begin{abstract}
Results from animal experiments showing that estradiol is neuroprotective were challenged 10 years ago by findings indicating an increased risk of dementia and stroke in women over 65 years of age taking conjugated equine estrogens. Our understanding of the complex signaling of estradiol in neural cells has recently clarified the causes of this discrepancy. New data indicate that estradiol may lose its neuroprotective activity or even increase neural damage, a situation that depends on the duration of ovarian hormone deprivation and on ageassociated modifications in the levels of other molecules that modulate estradiol action. These studies highlight the complex neuroprotective mechanisms of estradiol and suggest a window of opportunity during which effective hormonal therapy could promote brain function and cognition.
\end{abstract}

Neuroprotective actions of estradiol: myth or reality? By the end of the 20th century, numerous studies had demonstrated that $17 \beta$-estradiol exerts trophic actions on neurons and glial cells, promotes neuronal survival, and decreases neurodegenerative damage caused by a large variety of neuronal insults. However, in 2003 the findings of the Women's Health Initiative (WHI) study, a large, double-blind, placebo-controlled, randomized trial of hormone therapy (HT) in postmenopausal women, showed an increased risk of dementia and stroke in women over 65 years of age who received conjugated equine estrogens plus medroxyprogesterone acetate (MPA) compared to women who received placebo. In addition, HT did not prevent mild cognitive impairment [1]. This finding contradicted the evidence from animal models of neurodegenerative diseases and has introduced considerable confusion in the field.

Basic research in the post-WHI era has tried to identify possible causes of the discrepancies between laboratory studies and the WHI trial. The mechanisms involved in the neuroprotective actions of estradiol and other estrogens have been further explored in efforts to understand why HT may, in some cases, fail to promote neuroprotection. In particular, age and time after ovarian hormone deprivation when estradiol treatment is administered are factors that have been taken into consideration, especially because

Corresponding author: Garcia-Segura, L.M. (lmgs@cajal.csic.es) women in the WHI trial who took HT later in life had increased risks of dementia and stroke. These most recent findings underline the need to reconsider and further explore species-specific differences in the mechanism of estradiol in the brain and its neuroprotective role in human diseases of the central nervous system (CNS).

Neuroprotective actions of estradiol: basic research rebounded after the WHI trial challenge

Experimental evidence supporting neuroprotective actions of estradiol was so convincing before the WHI trial that the results of the trial in the long run did not lead to a decline in basic research on this topic. On the contrary, basic research in the post-WHI era has continued to accumulate evidence in support of neuroprotective actions of estradiol in animal models of human CNS disorders such as Alzheimer's disease [2], Parkinson's disease [3], stroke [4,5], multiple sclerosis [6] chronic hypertension [7] and traumatic brain injury [8], among others.

Recent evidence suggests that estradiol is not only protective, but may also activate restorative processes in the nervous system (Figure 1). Thus, estradiol protects neurons from brain ischemia first by reducing apoptosis and then by facilitating the incorporation of newly generated neurons from the subventricular zone to the ischemic regions [4]. An increase in hippocampal neurogenesis, mediated by brain-derived neurotrophic factor (BDNF), is also involved in the restorative action of estradiol in the hippocampus of hypertensive rats [7]. Furthermore, reparative actions of estradiol in the brain of adult male rats subjected to perinatal asphyxia have been detected [9].

Neuronal signaling pathways that mediate estradiolmediated neuroprotection

Classical estrogen receptors

The recent development of new selective agonists and antagonists for estrogen receptor (ER) $\alpha$-and $\beta$-mediated transcription has facilitated exploration of the role of classical ERs in the neuroprotective and neuroreparative mechanisms of estradiol, and has extended previous data obtained in ER $\alpha$ and ER $\beta$ knockout mice. Both ER $\alpha$ and $\mathrm{ER} \beta$ seem to be involved in the neuroprotective actions of the hormone, although they mediate the activation of different mechanisms and affect different parameters. 


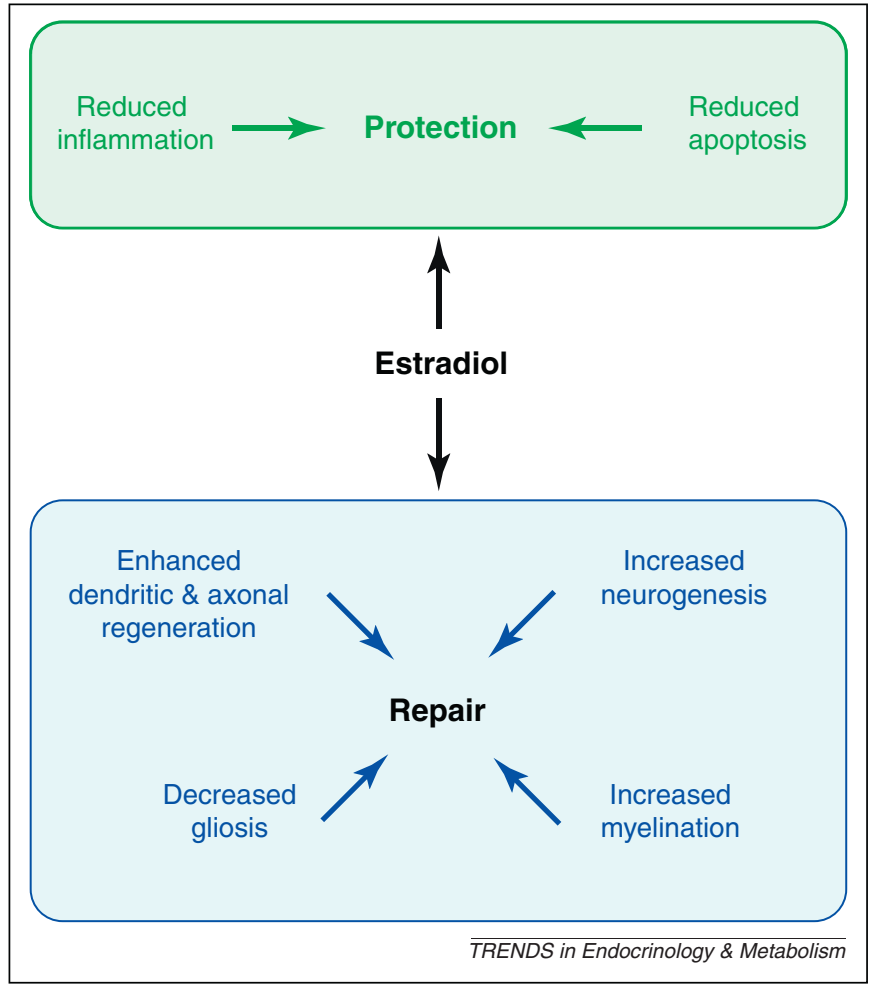

Figure 1. Estradiol activates both protective and reparative mechanisms after CNS injury. Estradiol exerts protective actions in the initial phases after CNS injury, and reduces inflammation and prevents apoptosis. In addition, estradiol promotes repair of injured neural tissue by enhancing neurogenesis, remyelination and regeneration of neuronal processes and by reducing reactive gliosis. Based on [4-10].

For instance, in experimental autoimmune encephalomyelitis, an ER $\alpha$ agonist was anti-inflammatory and exerted protection at the onset of the disease, whereas an ER $\beta$ agonist was not anti-inflammatory but induced recovery from the disease [10].

\section{Estradiol-induced membrane and cytoplasm signaling}

Numerous studies have shown the crucial importance of rapid and transient activation of mitogen-activated protein kinase (MAPK, ERK) signaling for the neuroprotective actions of estradiol. Activation of MAPK signaling is accompanied by activation of cAMP response element binding protein (CREB), induction of BCL2 expression, and inhibition of the apoptotic cascade [11,12]. The rapid neuroprotective effects induced by estradiol, such as transient activation of MAPK, may involve signaling mediated by non-classical ERs. One such candidate is Gprotein-coupled receptor 30 (GPR30). Although the role of GPR30 as a putative estrogen receptor is a matter of debate, recent studies have shown that a GPR30 agonist is able to imitate neuroprotective and neuritogenic actions of estradiol on hippocampal neurons [13-15]. Estradiolinduced rapid neuroprotective events may also involve classical ERs located in extranuclear compartments. Indeed, $\mathrm{ER} \alpha$ has been detected in the plasma membrane of neurons and astrocytes [16,17]. Caveolin-1 provides a scaffold for the interaction of $\mathrm{ER} \alpha$ with different membrane-associated molecules in neuronal membranes [18]. One of these molecules is a plasmalemmal voltage-dependent anion channel (VDAC) involved in amyloid- $\beta$-induced neurotoxicity. VDAC is rapidly phosphorylated by $17 \beta-$ estradiol through activation of protein kinase A (PKA) and Src-kinase, and this contributes to maintaining the channel in an inactive closed conformation [19]. Interestingly, in the cerebral cortex of Alzheimer's disease patients, the association of ER $\alpha$ and VDAC in lipid rafts is disrupted [20], which suggests that alterations in the function of $E R \alpha$ in the plasma membrane may be associated with neurodegenerative events.

\section{IGF1 and WNT signaling}

$\mathrm{ER} \alpha$ also interacts with membrane receptors, such as the insulin-like growth factor 1 (IGF1) receptor (IGF1R) [17]. In neurons, estradiol induces incorporation of $\mathrm{ER} \alpha$ into a macromolecular complex in which IGF1R and several components of IGF1R signaling are associated [21]. This molecular complex, which includes insulin receptor substrate-1 (IRS1), phosphoinositol 3 kinase (PI3K), AKT, glycogen synthase kinase $3 \beta$ (GSK3 $\beta$ ) and $\beta$-catenin, regulates neuronal survival [21]. PI3K activation results in phosphorylation and activation of AKT and, in turn, phosphorylation and inhibition of GSK3 $\beta$. Sustained activation of GSK3 $\beta$ is associated with neuronal death and hyperphosphorylation of tau. Estradiol, in synergism with IGF1, activates PI3K-AKT and inhibits GSK3 $\beta$ [21]. This hormonal action promotes neuroprotection, improves brain homeostasis [22] and reduces tau phosphorylation [23]. The interaction of estradiol and IGF1R in neuroprotection has been characterized in experimental models of excitotoxicity, Parkinson's disease and stroke [11,21,24-26]. Interestingly, inhibition of brain IGF1 signaling in these models resulted in attenuation of the neuroprotective effects of estradiol.

By modulating GSK3 $\beta$ activity, estradiol also regulates translocation of $\beta$-catenin to the cell nucleus and $\beta$ catenin-lymphoid enhancer binding factor-1 (LEF1)-mediated transcription [23]. Recent findings suggest that estradiol may also exert neuroprotective actions via modulation of WNT- $\beta$-catenin signaling. Specifically, in a model of global cerebral ischemia, estradiol downregulates expression of the neurodegenerative factor dickkopf-1 (DKK1), which is an antagonist of the WNT- $\beta$-catenin signaling pathway. DKK1 is involved in the neurodegeneration observed in a number of different pathologies, including Alzheimer's disease, epilepsy and stroke [27]. Estradiol promotes expression of WNT3 and translocation of $\beta$-catenin to the cell nucleus in the $\mathrm{CA} 1$ region of the hippocampus after global cerebral ischemia, and enhances expression of the anti-apoptotic factor survivin, a $\beta$-catenin-induced gene [27]. Administration of exogenous DKK1 abolishes the neuroprotective action of estradiol, the hormonal downregulation of tau hyperphosphorylation, and the hormonally induced $\beta$-catenin nuclear localization in this model [27].

\section{Seladin-1}

Another link between the neuroprotective mechanisms of estradiol and IGF1 is seladin-1 [28]. Seladin-1 (DHCR24) encodes the enzyme 3ß-hydroxysterol $\delta 24$-reductase, which catalyzes cholesterol synthesis from desmosterol. DHCR24 is an anti-apoptotic gene that is downregulated in 
several brain regions in Alzheimer's disease (for this reason it was named selective Alzheimer's disease indicator-1). In human neuroblasts, estradiol stimulates DHCR24 expression by directly binding to half-palindromic estrogen responsive elements in the promoter region of the DHCR24 gene [28]. In addition, estradiol stimulates synthesis of IGF1, which, through activation of IGF1R signaling, also increases DHCR24 expression [28]. The neuroprotective action of estradiol against $\beta$-amyloid toxicity in human neuroblasts is lost when DHCR24 is silenced [28]. DHCR24 is therefore an interesting gene that may represent a point of convergence between the neuroprotective actions of estradiol and IGF1 and the local neural synthesis of cholesterol and its metabolites, including neuroactive steroids.

\section{Neuroglobin and mitochondria}

Neuroglobin, a neuroprotective and anti-apoptotic protein, has been recently identified as a mediator of estrogenic neuroprotective actions. Estradiol induces expression of this globin in human neuroblastoma cells and in primary cultures of mouse hippocampal neurons through mechanisms involving ER $\beta$ [29]. Silencing of neuroglobin results in loss of the neuroprotective action that estradiol exerts against oxidative stress [29]. Further studies are necessary to determine the role of neuroglobin in the neuroprotective actions of estradiol in different in vivo models of neurodegenerative diseases. One possibility is that neuroglobin could be involved in estradiol-dependent neuroprotective effects by decreasing mitochondrial dysfunction caused by oxidative damage. Indeed, regulation of mitochondrial function and metabolism has emerged as one of the key actions exerted by estradiol to promote neuronal viability. The effect of estradiol on mitochondria involves direct actions on the mitochondrial genome, as well as regulation of nuclear transcriptional activity and modulation of membrane and cytoplasmic signaling [30].

\section{Non-neuronal cells contribute to estradiol-mediated neuroprotection}

In addition to activating protective mechanisms by direct actions on neurons expressing ERs, estradiol also activates complementary mechanisms in other cell types to promote neuroprotection and restoration of neural function (Figure 2). The importance of the neurovascular unit in the protective actions of estradiol against focal cerebral ischemia has recently been analyzed [4]. In addition to its actions on neurons and glial cells, estradiol interacts with other cell types such as endothelial cells, smooth muscle

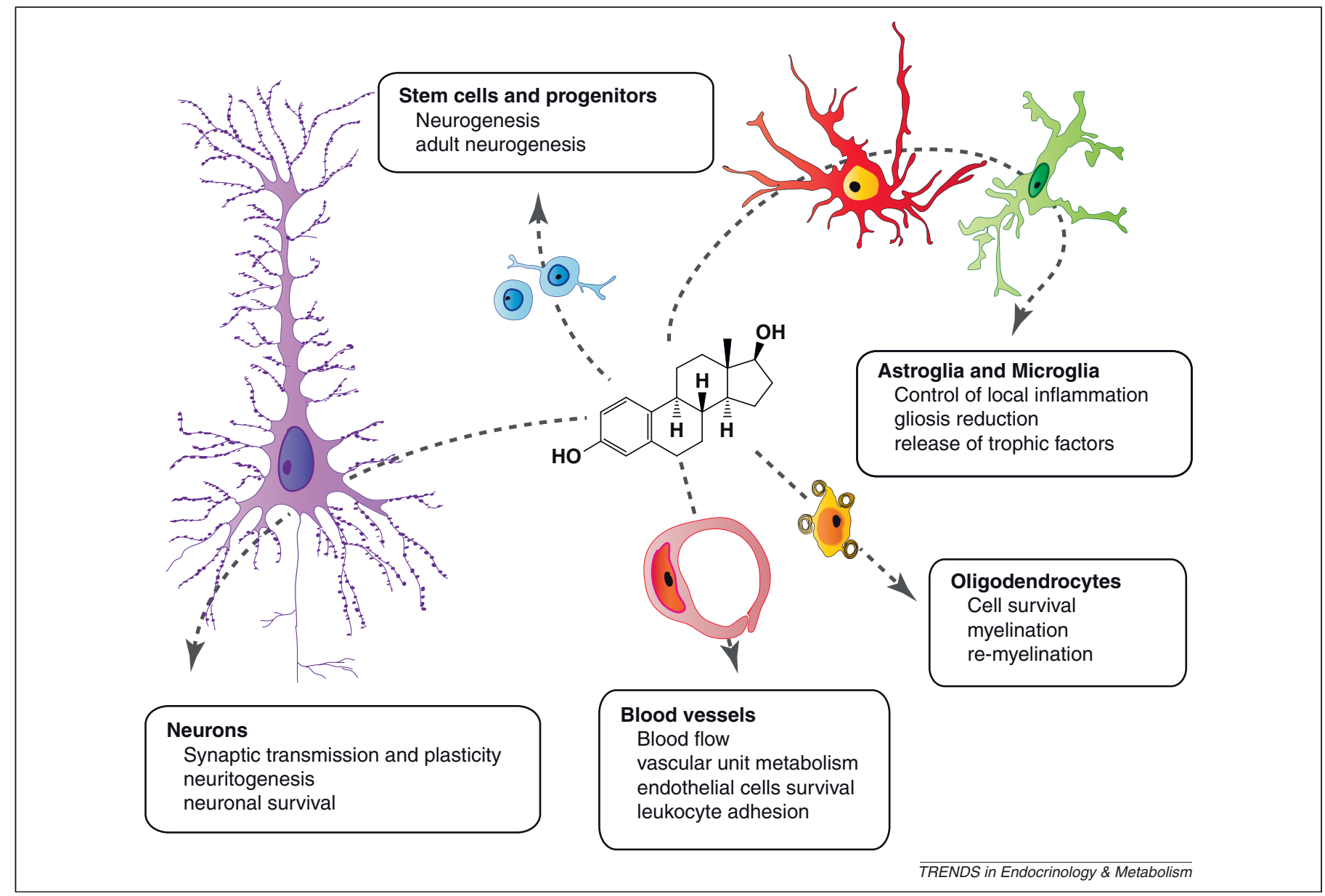

Figure 2. Estradiol triggers protective and reparative mechanisms acting on different cellular targets in the CNS. Estradiol acts on neurons expressing nuclear and membrane ERs to promote neuronal survival, neuritogenesis and synaptic function. Acting on stem cells and progenitors, estradiol regulates the generation of new neural cells, including neurons. In addition, the hormone reduces local inflammation of astrocytes and microglia and promotes neuronal survival through the release of growth factors from astrocytes. Estradiol also reduces oligodendrocyte cell loss and demyelination and facilitates remyelination. Actions of estradiol on endothelial cells, smooth muscle cells, platelets and leucocytes are also involved in hormonal control of the local inflammatory response and on the regulation of blood flow, metabolism of the neurovascular unit, leukocyte adhesion and endothelial cell survival. Based on [4,31-40]. 
cells, platelets and leukocytes to regulate cerebral blood flow, the metabolism of the neurovascular unit, the local inflammatory response, leukocyte adhesion and endothelial cell survival [4].

Direct or indirect actions of estradiol on microglia are important for the control of local inflammation [31,32]. Actions of estradiol on astrocytes also contribute to the control of inflammation and reactive gliosis, and to the response of neurons to neurodegenerative stimuli [33-35]. In this regard, it has recently been demonstrated that $\mathrm{ER} \alpha$ expressed by astrocytes mediates the neuroprotective action of estradiol in experimental autoimmune encephalomyelitis [36]. Recent findings suggest that actions of estradiol in the control of reactive gliosis involve cannabinoid receptors [37], an observation that opened the possibility of neuroprotective therapies based on the mechanisms of interaction of estradiol and cannabinoid signaling.

Another important cell type involved in the neuroprotective actions of estradiol is the oligodendrocyte. It is important to remember the considerable proportion of volume occupied by white matter in the human brain and the spinal cord, and the important role that white matter lesions have not only in demyelinating diseases, but also in stroke, traumatic CNS injury and other neurodegenerative diseases. Recent studies have investigated the actions of estradiol on oligodendrocytes and myelin integrity after different forms of white matter injury. The results have shown that estradiol is able to reduce, at least partially, oligodendrocyte cell loss and demyelination and to facilitate remyelination [38]. The protective actions of estradiol on oligodendrocytes and myelin may be mediated by the hormonal control of peripheral immune cells [39] and by direct actions on oligodendrocytes [40].

\section{Local estradiol synthesis and neuroprotection}

Enzymes for steroid synthesis and steroid metabolism, including aromatase, which is involved in the conversion of testosterone to estradiol, are expressed in the human brain [41]. Aromatase activity is rapidly regulated by neurotransmitters and by calcium-dependent phosphorylation. In turn, estradiol produced by aromatase is involved in the regulation of synaptic transmission, synaptic plasticity, adult neurogenesis and behavior [42-44]. In addition, aromatase expression is increased in the brain under pathological conditions such as after exocytotoxic lesions, traumatic injury or experimental stroke [45-48]. Several laboratories have found that aromatase expression in the brain of rodents and birds is neuroprotective and contributes to limiting neural damage after brain injury $[46,47]$. Therefore, it is tempting to speculate on the possible role of aromatase in the human brain. Aromatase expression in brain could compensate for the deficit in plasma estradiol levels caused by menopause. If this were the case, the brain of some perimenopausal women could be more adapted to the lack of peripheral estrogens than in others, depending on the level of local estradiol production. Furthermore, differences in brain aromatase expression may confer a different risk for the development of neurodegenerative diseases. Finally, brain aromatase may have potential implications with regard to HT in postmenopausal women.
In this case, it might be of interest to consider the possibility that estrogen administration may have a negative impact in brains that have found mechanisms (such as increased aromatase activity) to compensate for low peripheral hormonal levels by increasing local estradiol synthesis.

\section{Impact of natural progesterone and synthetic progestins on estrogenic neuroprotective actions}

Diverse progestins, synthetic molecules that have effects similar to progesterone, are frequently administered in combination with estrogens as HT in menopausal women to counteract the risk of endometrial cancer, which is associated with estrogen use when administered without a counterbalance of progestins. Therefore, it is important to determine the impact of progestins on estradiol-mediated neuroprotective mechanisms. The hormone progesterone is neuroprotective against excitotoxicity, stroke, traumatic brain and spinal cord injury, experimental multiple sclerosis, experimental immune encephalomyelitis and motoneuron degeneration [49-52]. However, not all the synthetic progestins are neuroprotective and not all of them have the same impact on the neuroprotective actions mediated by estradiol. For instance, MPA, the progestin used in the WHI study, is not only unable to exert neuroprotection [53], but also antagonizes the neuroprotective actions of estradiol on mitochondrial function [54]. By contrast, the combination of estradiol and progesterone is neuroprotective in rodent models of Parkinson disease [55], demyelinating diseases [56] and stroke [57], and protects serotonin neurons in a monkey model of surgical menopause [58]. However, recent studies suggest that natural progesterone may also interfere with some of the neuroprotective mechanisms of estradiol. Moreover, of crucial importance is the notion of continuous or cyclic progesterone delivery. Indeed, continuous progesterone delivery blocks the neuroprotective actions of estradiol in a rodent model of Alzheimer's disease, whereas cyclic progesterone administration enhances the neuroprotective action of estradiol in this model [59]. The interaction of progesterone and synthetic progestins with the neuroprotective mechanisms of estradiol needs to be further explored to fully understand and predict the neural outcome of combined HT.

\section{Experimental studies on animals and clinical data support a window of opportunity for HT}

In the WHI study, the majority of women started HT in the late postmenopausal stage (aged 65-79 years). By contrast, in observational studies showing a beneficial effect of HT on cognition, treatment was initiated in the perimenopausal period. This suggests that either age and/or time of HT initiation after natural or surgical menopause could influence the results. Current evidence suggests that early initiation of HT is associated with enhanced memory and beneficial effects on the brain [60,61]. In addition, a long period of treatment may also negatively affect the cognitive outcome of HT [62].

Recent experimental studies, showing that both age and previous duration of ovarian hormone deprivation affect the neuroprotective actions of estradiol, support 


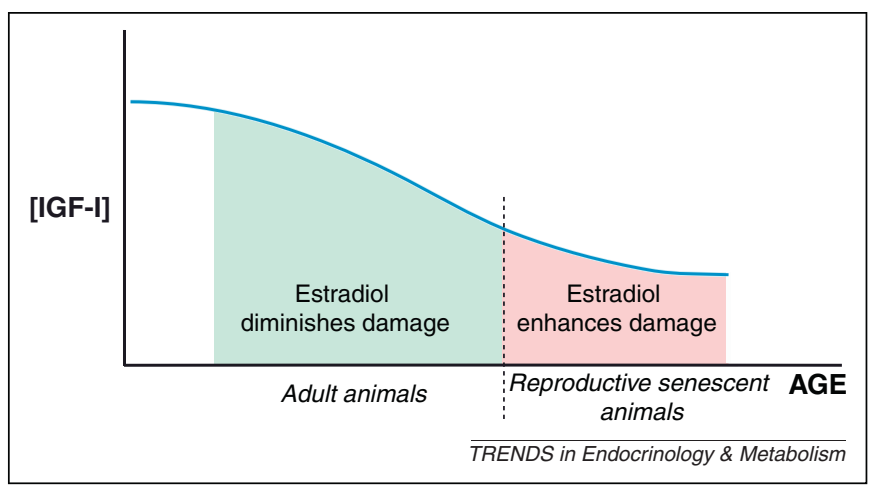

Figure 3. Estradiol shifts from being protective against experimental stroke in adult mature female rats to increasing damage in reproductive senescent females. Estradiol reduces brain damage induced by experimental stroke in adult female rats by a mechanism involving IGF-1 receptors. By contrast, estradiol increases brain damage after stroke in reproductive senescent animals, in which IGF-1 levels in plasma and brain tissue are decreased. The different outcome of estradiol therapy in adult and reproductive senescent females may explain why hormone therapy increases the risk of stroke in postmenopausal women whereas low-dose oral estrogen-based contraceptives do not affect the risk of stroke in young women. Based on $[24,65]$.

the concept of a window of opportunity for HT [23,63,64]. Thus, estradiol not only loses some of its neuroprotective actions in older animals, but may even shift from being neuroprotective in adult mature animals to being neurotoxic in reproductively senescent animals (Figure 3) [65]. This shift in outcome of estradiol therapy is associated with a decline in plasma IGF1 levels with aging [24], which further supports the importance of the interaction between estradiol and IGF1 and the neuroprotective mechanisms discussed above. These findings suggest that HT might have a different cognitive outcome in older women with low IGF1 plasma levels compared to younger women who may still have a moderate age-dependent decrease in IGF1 levels.

\section{Concluding remarks}

The studies reviewed here clearly support the neuroprotective functions of estradiol. However, there are many aspects that still need to be addressed, including determination of the best estrogenic formulations and whether cyclic versus continuous HT delivery is most suitable for promoting brain function and cognition, because it is now accepted that different estrogen and progesterone treatment regimens may have different impacts on the CNS $[58,66]$. In addition, it is important to understand the role that other factors, such as IGF1 and the different cellular and subcellular targets of estradiol, play in the neuroprotective mechanisms of the hormone. For instance, regulation of mitochondrial function and brain energy homeostasis by estradiol and its implications for neuroprotection are currently being investigated by several laboratories.

Another important consideration is that although estradiol is neuroprotective, its peripheral actions limit its use for the treatment or prevention of neurodegenerative diseases and affective disorders. Therefore, new alternatives to estradiol therapy based on the neuroprotective mechanisms of the hormone are being explored. Selective estrogen receptor modulators (SERMs), with agonistic estrogenic properties in the brain and antagonistic effects in tissues such as breast cancer cells, may represent an alternative to estradiol and may also be used in males. It has been shown that several SERMs are neuroprotective and reduce brain inflammation in different animal models of neurodegeneration [67]. SERMs also have positive effects on cognition and reduce anxiety-like and depression-like behaviors in rodents $[68,69]$. However, the effects of SERMs on brain function in humans have not been sufficiently explored [67,70].

The neuroprotective actions of other estrogenic compounds, including contraceptives [71], ligands for nonclassical estrogen receptors $[5,14]$ and non-feminizing estrogens [72], have been explored in animal models with promising results. Further developments may include the use of aromatase modulators to selectively increase brain estradiol synthesis as an alternative to the administration of estrogenic compounds. Given the importance of the interactions of estradiol and IGF1 neuroprotective mechanisms, the outcome of the modulation of IGF1 levels in parallel with administration of estrogenic therapies should also be explored in experimental neurodegenerative models.

\section{Acknowledgements}

We acknowledge support from BFU2008-02950-C03-01/02, PICT 2007 001044, CONICET PIP 00542 and UBA M016.

\section{References}

1 Shumaker, S.A. et al. (2003) Estrogen plus progestin and the incidence of dementia and mild cognitive impairment in postmenopausal women: the Women's Health Initiative Memory Study. J. Am. Med. Assoc. 289, 2651-2662

2 Pike, C.J. et al. (2009) Protective actions of sex steroid hormones in Alzheimer's disease. Front. Neuroendocrinol. 30, 239-258

3 Bourque, M. et al. (2009) Neuroprotective actions of sex steroids in Parkinson's disease. Front. Neuroendocrinol. 30, 142-157

4 Suzuki, S. et al. (2009) Neuroprotective effects of estrogens following ischemic stroke. Front. Neuroendocrinol. 30, 201-211

5 Etgen, A.M. et al. (2011) Neuroprotective actions of estradiol and novel estrogen analogs in ischemia: Translational implications. Front. Neuroendocrinol. 32, 336-352

6 Kipp, M. and Beyer, C. (2009) Impact of sex steroids on neuroinflammatory processes and experimental multiple sclerosis. Front. Neuroendocrinol. 30, 188-200

7 Pietranera, L. et al. (2010) Involvement of brain-derived neurotrophic factor and neurogenesis in oestradiol neuroprotection of the hippocampus of hypertensive rats. J. Neuroendocrinol. 22, 1082-1092

8 Barreto, G. et al. (2009) Selective estrogen receptor modulators decrease astrogliosis in the injured brain: effects of aging and prolonged depletion of ovarian hormones. Endocrinology 150, 5010-5015

9 Saraceno, G.E. et al. (2010) Estradiol therapy in adulthood reverses glial and neuronal alterations caused by perinatal asphyxia. Exp. Neurol. 223, 615-622

10 Tiwari-Woodruff, S. and Voskuhl, R.R. (2009) Neuroprotective and anti-inflammatory effects of estrogen receptor ligand treatment in mice. J. Neurol. Sci. 286, 81-85

11 Jover-Mengual, T. et al. (2007) MAPK signaling is critical to estradiol protection of CA1 neurons in global ischemia. Endocrinology 148, 1131-1143

12 Yang, L.C. et al. (2010) Extranuclear estrogen receptors mediate the neuroprotective effects of estrogen in the rat hippocampus. PLOS ONE 5 , e9851

13 Gingerich, S. et al. (2010) Estrogen receptor alpha and G-protein coupled receptor 30 mediate the neuroprotective effects of 17 beta-estradiol in novel murine hippocampal cell models. Neuroscience 170, 54-66

14 Lebesgue, D. et al. (2010) Acute administration of non-classical estrogen receptor agonists attenuates ischemia-induced hippocampal neuron loss in middle-aged female rats. PLoS ONE 5, e8642 
15 Ruiz-Palmero, I. et al. (2011) Notch/Neurogenin 3 signalling is involved in the neuritogenic actions of oestradiol in developing hippocampal neurones. J. Neuroendocrinol. 23, 355-364

16 Gorosito, S.V. et al. (2008) Estrogen receptor alpha is expressed on the cell-surface of embryonic hypothalamic neurons. Neuroscience 154, $1173-1177$

17 Dominguez, R. and Micevych, P. (2010) Estradiol rapidly regulates membrane estrogen receptor alpha levels in hypothalamic neurons. J. Neurosci. 30, 12589-12596

18 Marin, R. et al. (2009) Role of estrogen receptor alpha in membraneinitiated signaling in neural cells: interaction with IGF-1 receptor. J. Steroid Biochem. Mol. Biol. 114, 2-7

19 Herrera, J.L. et al. (2011) Estradiol and tamoxifen differentially regulate a plasmalemmal voltage-dependent anion channel involved in amyloid-beta induced neurotoxicity. Steroids 76, 840-844

20 Ramírez, C.M. et al. (2009) VDAC and ERalpha interaction in caveolae from human cortex is altered in Alzheimer's disease. Mol. Cell. Neurosci. 42, 172-183

21 Garcia-Segura, L.M. et al. (2010) Interactions of estradiol and insulinlike growth factor-I signalling in the nervous system: new advances. Prog. Brain Res. 181, 251-272

22 Alonso, A. et al. (2008) Chronic estradiol treatment improves brain homeostasis during aging in female rats. Endocrinology 149, 57-72

23 Varea, O. et al. (2010) Interaction of estrogen receptors with insulinlike growth factor-I and Wnt signaling in the nervous system. Steroids $75,565-569$

24 Selvamani, A. and Sohrabji, F. (2010) The neurotoxic effects of estrogen on ischemic stroke in older female rats is associated with age-dependent loss of insulin-like growth factor-1. J. Neurosci. 30, $6852-6861$

25 Quesada, A. et al. (2008) PI3 kinase/Akt activation mediates estrogen and IGF-1 nigral DA neuronal neuroprotection against a unilateral rat model of Parkinson's disease. Dev. Neurobiol. 68, 632-644

26 Lebesgue, D. et al. (2009) Estradiol rescues neurons from global ischemia-induced cell death: multiple cellular pathways of neuroprotection. Steroids 74, 555-561

27 Zhang, Q.G. et al. (2008) Role of Dickkopf-1, an antagonist of the Wnt/ beta-catenin signaling pathway, in estrogen-induced neuroprotection and attenuation of tau phosphorylation. J. Neurosci. 28, 8430-8441

28 Peri, A. et al. (2009) New insights on the neuroprotective role of sterols and sex steroids: the seladin-1/DHCR24 paradigm. Front. Neuroendocrinol. 30, 119-129

29 De Marinis, E. et al. (2011) 17ß-Estradiol - a new modulator of neuroglobin levels in neurons: role in neuroprotection against $\mathrm{H}_{2} \mathrm{O}_{2^{-}}$ induced toxicity. Neurosignals 18, 223-235

30 Simpkins, J.W. et al. (2010) Mitochondrial mechanisms of estrogen neuroprotection. Biochim. Biophys. Acta 1800, 1113-1120

31 Arevalo, M.A. et al. (2010) Actions of estrogens on glial cells: implications for neuroprotection. Biochim. Biophys. Acta 1800 , 1106-1112

32 Taylor, L.C. et al. (2010) 17beta-Estradiol protects male mice from cuprizone-induced demyelination and oligodendrocyte loss. Neurobiol. Dis. $39,127-137$

33 Carbonaro, V. et al. (2009) Enhanced expression of ERalpha in astrocytes modifies the response of cortical neurons to beta-amyloid toxicity. Neurobiol. Dis. 33, 415-421

34 Azcoitia, I. et al. (2010) Role of astroglia in the neuroplastic and neuroprotective actions of estradiol. Eur. J. Neurosci. 32, 1995-2002

35 Giraud, S.N. et al. (2010) Estradiol inhibits ongoing autoimmune neuroinflammation and NFkappaB-dependent CCL2 expression in reactive astrocytes. Proc. Natl. Acad. Sci. U.S.A. 107, 8416-8421

36 Spence, R.D. et al. (2011) Neuroprotection mediated through estrogen receptor $\alpha$ in astrocytes. Proc. Natl. Acad. Sci. U.S.A. 108, 8867-8872

37 López Rodríguez, A.B. et al. (2011) Estradiol decreases cortical reactive astrogliosis after brain injury by a mechanism involving cannabinoid receptors. Cereb. Cortex 21, 2046-2055

38 Crawford, D.K. et al. (2010) Oestrogen receptor beta ligand: a novel treatment to enhance endogenous functional remyelination. Brain 133 , 2999-3016

$39 \mathrm{Du}$, S. et al. (2011) Estrogen receptor- $\beta$ ligand treatment modulates dendritic cells in the target organ during autoimmune demyelinating disease. Eur. J. Immunol. 41, 140-150
40 Hirahara, Y. et al. (2009) The localization and non-genomic function of the membrane-associated estrogen receptor in oligodendrocytes. Glia $57,153-165$

41 Azcoitia, I. et al. (2011) Estradiol synthesis within the human brain. Neuroscience 191, 139-147

42 Remage-Healey, L. et al. (2010) Brain estrogens rapidly strengthen auditory encoding and guide song preference in a songbird. Proc. Natl. Acad. Sci. U.S.A. 107, 3852-3857

43 Zhou, L. et al. (2010) Aromatase inhibitors induce spine synapse loss in the hippocampus of ovariectomized mice. Endocrinology 151, $1153-1160$

44 Charlier, T.D. et al. (2010) Diversity of mechanisms involved in aromatase regulation and estrogen action in the brain. Biochim. Biophys. Acta 1800, 1094-1105

45 Gatson, J.W. et al. (2011) Aromatase is increased in astrocytes in the presence of elevated pressure. Endocrinology 152, 207-213

46 Saldanha, C.J.et al. (2009) Neuroprotective actions of brain aromatase. Front. Neuroendocrinol. 30, 106-118

47 Garcia-Segura, L.M. (2008) Aromatase in the brain: not just for reproduction anymore. J. Neuroendocrinol. 20, 705-712

48 Pietranera, L. et al. (2011) Increased aromatase expression in the hippocampus of spontaneously hypertensive rats: effects of estradiol administration. Neuroscience 174, 151-159

49 De Nicola, A.F. (2009) Progesterone neuroprotection in traumatic CNS injury and motoneuron degeneration. Front. Neuroendocrinol. $30,173-187$

50 Stein, D.G. and Wright, D.W. (2010) Progesterone in the clinical treatment of acute traumatic brain injury. Expert. Opin. Invest. Drugs 19, 847-857

51 Frye, C.A. and Walf, A. (2011) Progesterone, administered before kainic acid, prevents decrements in cognitive performance in the Morris Water Maze. Dev. Neurobiol. 71, 142-152

52 Moralí, G. et al. (2011) Neuroprotective effects of progesterone and allopregnanolone on long-term cognitive outcome after global cerebral ischemia. Restor. Neurol. Neurosci. 29, 1-15

53 Ciriza, I. et al. (2006) Reduced metabolites mediate neuroprotective effects of progesterone in the adult rat hippocampus. The synthetic progestin medroxyprogesterone acetate (Provera) is not neuroprotective. $J$. Neurobiol. 66, 916-928

54 Irwin, R.W. et al. (2011) Medroxyprogesterone acetate antagonizes estrogen up-regulation of brain mitochondrial function. Endocrinology $152,556-567$

55 Morissette, M. et al. (2008) Estrogen and SERM neuroprotection in animal models of Parkinson's disease. Mol. Cell. Endocrinol. 290, $60-69$

56 Acs, P. et al. (2009) 17beta-Estradiol and progesterone prevent cuprizone provoked demyelination of corpus callosum in male mice. Glia 57, 807-814

57 Dang, J. et al. (2011) Gonadal steroids prevent cell damage and stimulate behavioral recovery after transient middle cerebral artery occlusion in male and female rats. Brain Behav. Immun. 25, 715-726

58 Lima, F.B. and Bethea, C.L. (2010) Ovarian steroids decrease DNA fragmentation in the serotonin neurons of non-injured rhesus macaques. Mol. Psychiatry 15, 657-668

59 Carroll, J.C. et al. (2010) Continuous and cyclic progesterone differentially interact with estradiol in the regulation of Alzheimerlike pathology in female $3 \times$ transgenic-Alzheimer's disease mice. Endocrinology 151, 2713-2722

60 Maki, P.M. et al. (2011) Perimenopausal use of hormone therapy is associated with enhanced memory and hippocampal function later in life. Brain Res. 1379, 232-243

61 Rocca, W.A. et al. (2011) Oophorectomy, menopause, estrogen treatment, and cognitive aging: clinical evidence for a window of opportunity. Brain Res. 1379, 188-198

62 Hogervorst, E. and Bandelow, S. (2010) Sex steroids to maintain cognitive function in women after the menopause: a meta-analyses of treatment trials. Maturitas $66,56-71$

63 Suzuki, S. et al. (2007) Timing of estrogen therapy after ovariectomy dictates the efficacy of its neuroprotective and antiinflammatory actions. Proc. Natl. Acad. Sci. U.S.A. 104, 6013-6018

64 Hamilton, R.T. et al. (2011) Hippocampal responsiveness to $17 \beta-$ estradiol and equol after long-term ovariectomy: Implication for a therapeutic window of opportunity. Brain Res. 1379, 11-22 
65 Selvamani, A. and Sohrabji, F. (2010) Reproductive age modulates the impact of focal ischemia on the forebrain as well as the effects of estrogen treatment in female rats. Neurobiol. Aging 31, $1618-1628$

66 Strom, J.O. et al. (2010) Different methods for administering 17betaestradiol to ovariectomized rats result in opposite effects on ischemic brain damage. BMC Neurosci. 11, 39

67 Arevalo, M.A. et al. (2011) Selective estrogen receptor modulators as brain therapeutic agents. J. Mol. Endocrinol. 46, R1-R9

68 Lagunas, N. et al. (2011) Estrogen receptor ligands counteract cognitive deficits caused by androgen deprivation in male rats. Horm. Behav. 59, 581-584
69 Walf, A.A. and Frye, C.A. (2010) Raloxifene and/or estradiol decrease anxiety-like and depressive-like behavior, whereas only estradiol increases carcinogen-induced tumorigenesis and uterine proliferation among ovariectomized rats. Behav. Pharmacol. 21, 231-240

70 Espeland, M.A. et al. (2010) Relative effects of tamoxifen, raloxifene, and conjugated equine estrogens on cognition. J. Womens Health (Larchmt.) 19, 371-379

71 Picazo, O. et al. (2010) Neuroprotective actions of the synthetic estrogen 17alpha-ethynylestradiol in the hippocampus. Cell. Mol. Neurobiol. 30, 675-682

$72 \mathrm{Yi}$, K.D. et al. (2011) The assessment of non-feminizing estrogens for use in neuroprotection. Brain Res. 1379, 61-70 